Archived version from NCDOCKS Institutional Repository http://libres.uncg.edu/ir/asu/

\title{
Appalachľan
}

B O O N E, N O R T H C A R O L I N A

\section{Telepractice Versus In-Person Delivery Of Voice Therapy For Primary Muscle Tension Dysphonia}

\author{
By: Balaji Rangarathnam, Gary H. McCullough, Hylan Pickett, Richard I. \\ Zraick, Ozlem Tulunay-Ugur, and Kimberly C. McCullough
}

\begin{abstract}
Purpose: The purpose of this study was to investigate the utility of telepractice for delivering flow phonation exercises to persons with primary muscle tension dysphonia (MTD). Method: Fourteen participants with a diagnosis of primary MTD participated, 7 on site and 7 at remote locations. Each participant received 12 treatment sessions across 6 weeks. Treatment consisted of flow phonation voice therapy exercises. Auditory-perceptual, acoustic, aerodynamic, and quality-of-life measures were taken before and after treatment. Results: Perceptual and qualityof-life measures were significantly better posttreatment and were statistically equivalent across groups. Acoustic and aerodynamic measures improved in both groups, but changes did not reach statistical significance. Results for the 2 service delivery groups were comparable, with no significant differences observed for perceptual and quality-of-life measures. Conclusions: Although the American Speech-Language-Hearing Association supports the use of telepractice for speech-language pathology services, evidence for the use of telepractice for providing behavioral treatment to patients with MTD has been lacking. The results of this study indicate that flow phonation exercises can be successfully used for patients with MTD using telepractice.
\end{abstract}

Balaji Rangarathnam, Gary H. McCullough, Hylan Pickett, Richard I. Zraick, Ozlem Tulunay-Ugur, and Kimberly C. McCullough (2015) "Telepractice Versus In-Person Delivery Of Voice Therapy For Primary Muscle Tension Dysphonia" American Journal of Speech-Language Pathology vol. 24 pp. 386-399

[doi:10.1044/2015_AJSLP-14-0017] Version of Record Available At www.asha.org 
$\mathrm{T}$ he American Speech-Language-Hearing Association (ASHA; 2002) defines telepractice as the application of telecommunications technology to delivery of professional services at a distance by linking clinician to client, or clinician to clinician, for assessment, intervention, and/or consultation. Moreover, ASHA emphasizes that telepractice is an appropriate model of service delivery for the professions of speech-language pathology and audiology and may be used to overcome barriers of access to services caused by distance, unavailability of specialists and/or subspecialists, and impaired mobility. With the growth of populations requiring services and a relative dearth of service providers in rural areas, telepractice is becoming more commonplace in health-care service delivery. Research evidence to support the efficacy of the use of telepractice is growing but not extensive. It is important to define the utility of telepractice in general, but it is also important to focus on specific types of treatments for specific types of patients. Telepractice may be more effective for one population or one type of treatment than another. In this study, we investigated the use of a flow phonation voice treatment method for individuals with primary muscle tension dysphonia (MTD), delivered through telepractice.

\section{Telepractice and Voice Treatment}

There have been reports of successful outcomes with delivery of voice treatment using telepractice. Mashima, Birkmire-Peters, Holtel, and Syms (1999) randomly assigned 10 patients to either a control condition $(n=4)$, in which therapy was conducted on site, or to an experimental condition $(n=6)$, in which the therapy was offered through telepractice. Patients were assessed using laryngoscopy, perceptual and acoustic voice measures, and patient-reported treatment outcomes. The results indicated that posttreatment recordings on all four measures were rated better than pretreatment recordings for the telepractice group, whereas 
voice recordings of only four of the six participants in the in-person group were rated as improved posttreatment. The findings were reported as preliminary results in an article addressing telepractice principles for speech-language pathology. Details about the voice therapy approaches used were not provided.

Extending upon their previous work, Mashima et al. (2003) compared voice treatment delivered in person and through telepractice for two groups of individuals with laryngeal conditions, including nodules, vocal fold paralysis, hyperfunction, and edema. Telepractice and in-person groups consisted of 23 and 28 individuals, respectively. Participants were assigned to the groups randomly but were matched for diagnostic category. Patient-specific treatment approaches were followed. Depending on the vocal pathology, facilitating voice treatment approaches (Boone, McFarlane, Von Berg, \& Zraick, 2013) - that is, focus, pitch adjustment, yawn-sigh, easy onset, open-mouth approach, pitch inflections, and chant talk-“confidential voice" (Colton \& Casper, 1990) or vocal function exercises (Stemple, Glaze, \& Gerdeman, 2000) were used. Vocal hygiene education was provided to all participants in both groups as well. Perceptual and acoustic voice assessments, patient satisfaction, and laryngoscopic images were compared before and after treatment for both groups. Posttreatment gains were shown in both groups and were comparable, implying that voice treatment delivered through telepractice was as effective as in-person treatment for these participants.

Tindall, Huebner, Stemple, and Kleinert (2008) examined voice treatment outcomes delivered through telepractice for a group of individuals with Parkinson's disease and compared those from a previously reported study (Ramig, Sapir, Fox, \& Countryman, 2001) on individuals who were treated in in-person sessions. Twenty-four individuals diagnosed with idiopathic Parkinson's disease were provided the Lee Silverman Voice treatment (LSVT) approach through video phone calling. Significant improvement in vocal intensity post therapy was reported, and the results were in good agreement with those obtained by Ramig et al. (2001) for in-person therapy.

Constantinescu et al. (2010a, 2010b, 2011) reported a series of studies demonstrating the utility of telepractice in voice assessment and treatment of individuals with Parkinson's disease. In their first study (Constantinescu et al., 2010a), the authors examined the validity of assessing speech and voice in 61 individuals with Parkinson's disease in telepractice versus in-person sessions and reported comparable results for vocal sound pressure level, phonation time, pitch range, sentence intelligibility, and communication efficiency in reading. Furthermore, the authors reported a case report (Constantinescu et al., 2010b) validating the utility of online delivery of LSVT. A patient with idiopathic Parkinson's disease was treated remotely for voice and speech impairments using LSVT and showed improvements in sound pressure levels, duration of sustained vowel production, vocal quality, and intelligibility, as well as high satisfaction with the treatment. The authors also reported successful delivery of the LSVT in 34 participants with Parkinson's disease and hypokinetic dysarthria (Constantinescu et al., 2011). They reported noninferiority of the online delivery as compared to in-person delivery on the basis of a noninferiority analysis of the data; that is, effects of the online delivery were not worse than the effects of in-person delivery by more than a specified margin.

The aforementioned studies suggest that voice treatment delivered through telepractice can be as effective as treatment delivered in person. Although the primary goal of the aforementioned studies by Mashima and colleagues $(1999,2003)$ was to investigate the efficacy of telepractice, control for participant homogeneity would add value to research related to voice therapy and telepractice. Research in the online delivery of LSVT to individuals with Parkinson's disease has been shown to be useful. However, research demonstrating the efficacy of LSVT for improving vocal hypofunction in patients with Parkinson's using telepractice does not essentially mean that other behavioral voice therapies, such as therapies for vocal hyperfunction, can be delivered successfully via telepractice. It would be useful to establish the efficacy of various types of treatments used with telepractice before advocating routine clinical use.

\section{$M T D$}

MTD has been defined as a behaviorally based voice disorder (Morrison \& Rammage, 1993) characterized by "imbalanced" laryngeal or perilaryngeal muscle activity that putatively involves vocal fold hyperfunction, laryngeal constriction, or bowing (Altman, Atkinson, \& Lazarus, 2005; Mathieson et al., 2009). MTD could be primary or secondary. Primary $M T D$ is defined as a voice problem in the absence of known concurrent structural or neurologic abnormalities (Awan \& Roy, 2009; Roy, 2003; Verdolini, Rosen, \& Branski, 2006). Secondary MTD is defined as a response to organic conditions and may affect voice quality, pitch, or loudness (Mathieson et al., 2009; Van Houtte, Van Lierde, \& Claeys, 2011). Although there is no internationally accepted classification system, endoscopic assessment typically reveals constriction of the laryngeal mechanism in one or more of the following ways: (a) anterior-posterior constriction of the vocal folds, (b) lateral-medial constriction of the vocal folds, and/or (c) approximation of the ventricular or false vocal folds. When severe enough, a sphincter-like closure of the entire larynx occurs (Rubin, Sataloff, \& Korovin, 2006).

MTD, whether primary or secondary, alters phonatory airflow substantially. It would be simplest if increased "tension" equaled vocal fold hyperadduction, increased laryngeal resistance to airflow (Rlaw), and decreased phonatory airflow, but this is not the universal case. Higgins, Chait, and Schulte (1999) reported large interparticipant variations in aerodynamic characteristics of individuals with primary and secondary MTD. Five aerodynamic profiles were recently identified in women with primary MTD as compared to women with normal voices (Gillespie, GartnerSchmidt, Rubinstein, \& Abbott, 2013). These profiles were (a) normal flow, normal estimated subglottic pressure (est-Psub); (b) high flow, high est-Psub; (c) low flow, normal 
est-Psub; (d) normal flow, high est-Psub; and (e) high flow, normal est-Psub. Estimated subglottic pressure can increase as Rlaw increases. It is not feasible to establish a one-to-one correlation between phonatory airflow and a particular pattern of laryngeal constriction/hyperfunction.

Voice therapy is considered the primary choice of treatment for improving the perceptually abnormal voice caused by primary or secondary MTD (Roy, 2008). The goal of voice therapy is to bring about relaxation of the ineffectively tensed musculature and improve vocal quality. Various muscle "rebalancing" approaches and research data supporting their use have been proposed over the years. Some of these include vocal function exercises (Stemple, Glaze, \& Gerdeman, 2000), resonant voice therapy (Verdolini-Marston, Burke, Lessac, Glaze, \& Caldwell, 1995), the accent method (Kotby, 1995), voice facilitating approaches (Boone et al., 2013), and circumlaryngeal massage (Roy, Ford, \& Bless, 1996; Roy \& Leeper, 1993). One treatment method that has been used in voice clinics is the flow phonation method, initially proposed by Stone and Casteel (1982). Flow phonation emphasizes the channeling of the airstream using a slightly abducted laryngeal position, which eventually facilitates clear vocal quality. The approach uses exercises, such as blowing bubbles in a cup of water, gargling, and blowing air into tissue paper, to facilitate airflow through a relaxed, balanced, and open vocal tract (Gartner-Schmidt, 2010; Stone \& Casteel, 1982). Variants of these exercises have been used with reported clinical success (Gartner-Schmidt, 2010). McCullough et al. (2012) recently provided the first data-based study of this method's utility for improving airflow and decreasing symptoms of vocal hyperfunction. The authors reported data from six participants who were treated for primary MTD using flow phonation exercises for a 6-week period. Patients were assessed on acoustic and perceptual measures as well as the Voice Handicap Index (Jacobson et al., 1997) before and after treatment. A Phonatory Aerodynamic System (PAS; KayPENTAX) was also used to measure phonatory airflow (i.e., flow of air through the larynx during phonation) and Rlaw, which is resistance to the flow of air at the level of the vocal folds, before and after treatment as well as in every therapy session. In addition to improvements in perceptual and acoustic measures of voicing and voice handicap ratings, most of the patients with primary MTD who completed the flow phonation exercise protocol decreased Rlaw values (i.e., laryngeal resistance) and increased phonatory airflow. Given the apparent utility of this method in alleviating vocal signs and symptoms, as demonstrated by McCullough et al., it is of interest to determine if the same method can be used with a telepractice delivery model. The current investigation served to determine the utility of telepractice for providing this type of treatment to individuals with primary MTD.

With the prevalence of a disorder such as MTD, millions of people are affected. For example, Cohen, Kim, Roy, Asche, and Courey (2012b) reported about 536,943 individuals among 55 million individuals in a national database of patients treated by primary care physicians and otolaryngologists received a diagnosis of dysphonia. In a similar study with the same database, Cohen, Kim, Roy, Asche, and Courey (2012a) reported that nearly three fourths of the population with a diagnosis of dysphonia were referred for medical treatment across a $12-$ month period. MTD is a significant cause of dysphonia. Data from various studies (Roy, 2003; Sama, Carding, Price, Kelly, \& Wilson, 2001) indicate that the caseload of primary MTD in voice clinics can range from $40 \%$ to $60 \%$, implying that it is one of the most common voice disorders seen in voice clinics. Outside of regional voice clinics, this type of treatment may not be available. Patients currently requiring voice treatment often travel long distances to metropolitan areas with more advanced rehabilitation facilities. Traveling two or three times per week for treatment imposes great hardships. Developing a way to provide treatment to such patients closer to home could greatly improve quality of care and quality of life, reducing the burdens imposed by time off from work and costs of travel. A high percentage of people (65\%) are reported to drop out of voice treatment (Hapner, PortoneMaira, \& Johns, 2009). The interval between voice therapy referral and scheduling voice therapy has been reported as an important variable affecting adherence to voice therapy (Portone-Maira, Wise, Johns, \& Hapner, 2011). Telepractice will offer an opportunity to decrease this time interval for individuals who do not have easier access to clinics, thereby reducing dropout from treatment (Tindall, 2012). Moreover, telepractice provides a "greener" method for treating patients. Less travel means reductions in greenhouse gases and smaller carbon footprints on the planet (Masino, Rubinstein, Lem, Purdy, \& Rossos, 2010).

Telepractice offers the potential to extend clinical services to remote, rural, and underserved populations and to culturally and linguistically diverse populations as well. A health care survey of speech-language pathologists across health care settings conducted by ASHA in 2009 indicated that $25 \%$ of the respondents had unfilled positions in their facility. The highest percentage of vacancies $(36 \%)$ was in home health (ASHA, 2009). Although the rate of reported vacancies of speech-language pathologists in health care has decreased from its high of 40\% in 2005 (ASHA, 2005), shortages are most likely to be felt in rural and underserved areas. Telepractice offers one solution to all these issues. Although the need for telepractice has been realized, actual clinical practice is relatively sparse. An ASHA survey (2002) reported that only $2 \%$ of 1,667 professionals who participated in the survey were actively involved in research or clinical care using telepractice. This number has certainly improved over the past decade, but there is a need to develop, validate, and promote assessment/treatment protocols for telepractice. Therefore, this study was conducted with the primary objective of determining the utility of telepractice for delivering the flow phonation voice treatment method to individuals with primary MTD. We hypothesized that patients treated via telepractice would demonstrate improvements in phonatory airflow and Rlaw as well as improvements in acoustic and perceptual measures and voice handicap equivalent to those receiving in-person evaluation and treatment. 


\section{Method}

The study had a prospective randomized control design. Randomization was done to eliminate bias and confounding in treatment assignment. Fourteen (11 women, three men) participants (see Table 1) were evaluated in person at an outpatient voice and swallowing clinic of a university medical center and were randomly assigned, via a computer-generated random numbers table, to receive treatment either in person or via telepractice at an affiliated outreach facility. The participants were 16 years and older and presented with "muscle tension dysphonia" documented in their medical record by an otolaryngologist, as discussed shortly. All participants had primary MTD, and those who presented with organic vocal lesions, head and neck cancer, spasmodic dysphonia or other neurological disorders, respiratory disorders including asthma, or oropharyngeal dysphagia were excluded from the study. In addition, participants who used other voice therapy methods at a different facility or pharmacological treatment (other than proton pump inhibitors recommended for disorders of laryngopharyngeal reflux-related symptoms) for the voice problem were excluded from the study. Baseline characteristics of the participants are reported in Table 2 . The study was approved by the medical center's institutional review board, and all participants provided written informed consent.

\section{Procedures}

\section{Assessment}

Laryngoscopic assessment. Laryngoscopic assessment was performed either by the study otolaryngologist (the fifth author) or the first author. A flexible naso-endoscope was inserted into one of the nares, depending on patient preference, after application of topical anesthesia $(0.2 \mathrm{ml}$ viscous lidocaine delivered on cotton-tip applicator). Once a clear image of the larynx was obtained, the patient was asked to sustain /i/ for at least $3 \mathrm{~s}$ at his or her most comfortable speaking pitch and loudness as well as at his or her lowest pitch and highest pitch, increasing and decreasing loudness generically. Vocal fold function and anatomy were examined in regular halogen mode and with stroboscopy. The otolaryngologist and the first author reviewed videostroboscopic examinations to ensure proper fit with requirements of primary MTD. MTD was defined visually as one of the four types described by Rubin et al. (2006) and further characterized according to criteria established by Koufman and Blalock (1982); that is, laryngoscopic studies for each patient were examined for the presence of lateralmedial compression, anterior-posterior compression, and ventricular adduction/supraglottic compression. Lateralmedial compression was identified as hyperadduction of the vocal folds in the lateral medial axis. Anterior-posterior compression was identified as constriction of the vocal tract at the level of the laryngeal vestibule in the anteriorposterior axis. Ventricular adduction/supraglottic compression was identified as supraglottic muscle activity, including partial or complete adduction of false vocal folds. All the participants presented with at least one of these findings. Patients with organic conditions were excluded. The otolaryngologist and a speech-language pathologist arrived at a consensus on the presence of MTD through running discussions.

Auditory-perceptual assessment. Assessment procedures were carried out within 2 weeks of an established diagnosis. Auditory-perceptual assessment was conducted using the Consensus Auditory Perceptual Evaluation of Voice (CAPE-V; Kempster, Gerratt, Verdolini Abbott, Barkmeier-Kramer, \& Hillman, 2009; Zraick et al., 2011). This assessment provides a measure of the clinician's perceptual ratings of a patient's voice on a variety of parameters, including breathiness, roughness, strain, pitch, loudness, and overall severity. Voice samples consisted of sustained /a/ and /i/ for at least $3 \mathrm{~s}$, reading of six sentences, and spontaneous speech. All voice samples were rated at the beginning and the end of treatment by a speech-language pathologist

Table 1. Demographic characteristics of study participants.

\begin{tabular}{|c|c|c|c|c|c|c|}
\hline Participant & Age & Gender & Group & Occupation & $\begin{array}{l}\text { Days post-onset } \\
\text { of symptoms }\end{array}$ & Additional information \\
\hline TVS001 & 53 & $\mathrm{~F}$ & Telepractice & College professor & 4 months & - \\
\hline TVS002 & 81 & M & In person & Retired real estate agent & 4 months & - \\
\hline TVS003 & 56 & $\mathrm{~F}$ & Telepractice & Retired teacher & 3 months & Prior history of thyroidectomy \\
\hline TVS006 & 57 & M & In person & Real estate agent & 4 months & Medications for reflux symptoms \\
\hline TVS011 & 33 & $\mathrm{~F}$ & In person & Business manager & 1 month & - \\
\hline TVS014 & 56 & $\mathrm{~F}$ & Telepractice & Part-time real estate agent & 2 years & - \\
\hline TVS015 & 59 & $\mathrm{~F}$ & In person & Radio and media & 5 months & - \\
\hline TVS016 & 39 & $\mathrm{~F}$ & Telepractice & Business manager & 2 months & Medications for reflux symptoms \\
\hline TVS018 & 51 & $\mathrm{~F}$ & Telepractice & Nurse & 1 month & - \\
\hline TVS021 & 60 & $\mathrm{~F}$ & In person & $\begin{array}{l}\text { Unemployed during the initial } \\
\text { assessment; previously a clerk }\end{array}$ & 3 years & 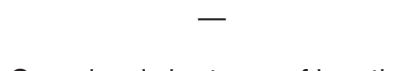 \\
\hline TVS022 & 59 & $\mathrm{~F}$ & Telepractice & Nurse & 3 months & Occasional shortness of breath \\
\hline TVS023 & 16 & $\mathrm{~F}$ & In person & School student & 8 months & - \\
\hline TVS024 & 60 & $\mathrm{~F}$ & Telepractice & Retired teacher & 1 year & - \\
\hline TVS026 & 32 & M & In person & Business manager & 2 months & - \\
\hline
\end{tabular}

Note. $\mathrm{F}=$ female; $\mathrm{M}=$ male. 
Table 2. Baseline characteristics of study participants.

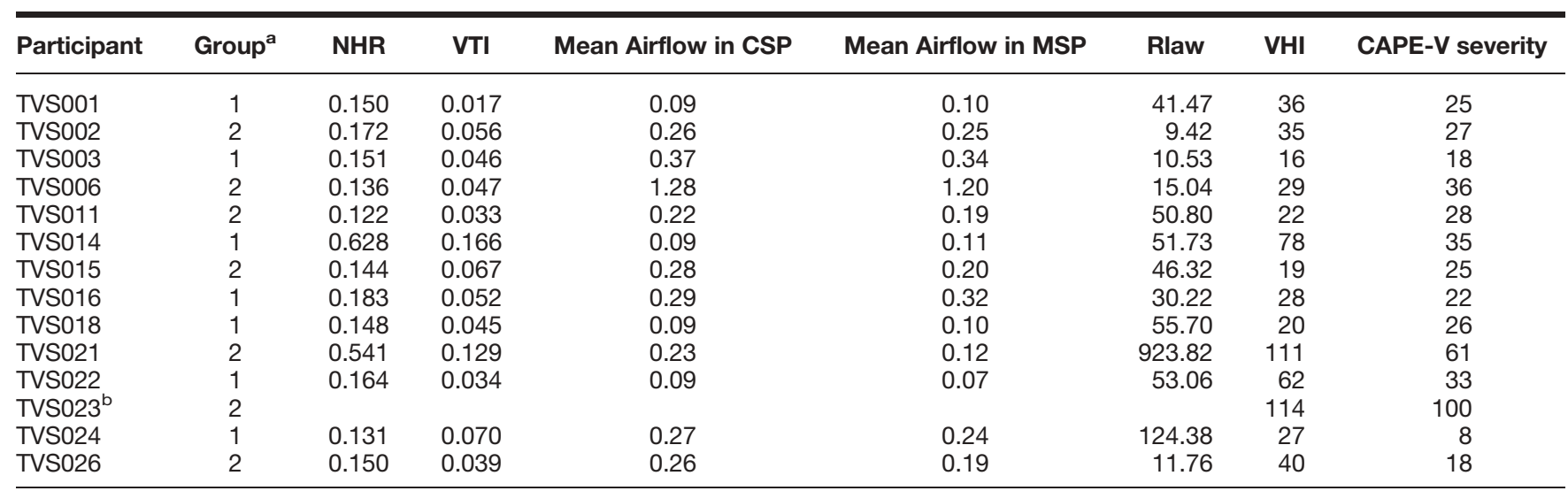

Note. $\mathrm{NHR}=$ noise-to-harmonic ratio; $\mathrm{VTI}=$ voice turbulence index; $\mathrm{CSP}=$ comfortable sustained phonation; $\mathrm{MSP}=$ maximum sustained phonation; Rlaw = laryngeal resistance; VHI = Voice Handicap Index; CAPE-V = Consensus Auditory Perceptual Evaluation of Voice.

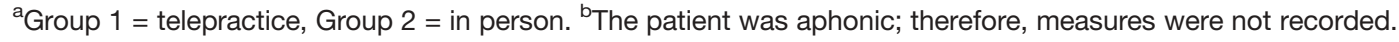

(the second author), and then $10 \%$ of the data were rerated blindly in random order by two other speech-language pathologists (the fourth author and another speech-language pathologist), both with extensive experience in evaluation of voice disorders. Pre- and posttreatment ratings for each participant were made together, and the conditions of time point and participant information were blinded; that is, preand posttreatment samples of each participant's voice were provided to each rater with order of occurrence randomized. Thus, each rater had two samples to rate without the knowledge of whether they had been obtained prior to or after treatment. Only the overall severity measure was considered for analysis purposes.

Acoustic assessment. Acoustic measures were made from recordings on the KayPENTAX Computerized Speech Lab 4500 (KayPENTAX, Inc.) in a room with nominal ambient noise. The default calibration settings of the Computerized Speech Lab Model 4500 were used, and the microphone (Shure SM48) was kept at a consistent distance of approximately 6 in. from the speaker's mouth. For the multidimensional voice profile analysis, the voice sample consisted of an /a/ vowel sustained for $5 \mathrm{~s}$, which was then analyzed for noise-to-harmonic ratio (NHR), voice turbulence index (VTI), and frequency and intensity perturbation measures. NHR has been demonstrated to correlate well with perceptual roughness (Bhuta, Patrick, \& Garnett, 2004; de Krom, 1995), whereas VTI represents the turbulence caused by incomplete adduction of the vocal folds (Di Nicola, Fiorella, Spinelli, \& Fiorella, 2006).

Aerodynamic measurement. Aerodynamic assessment of phonatory airflow and Rlaw were derived using the PAS Model 6600 (KayPENTAX). The following tasks were conducted:

(a) Maximum sustained phonation (MSP): Participants were instructed to take a deep breath, then to produce a sustained open vowel (/a/) at a comfortable pitch and loudness for as long as they could sustain voicing in one breath.

(b) Comfortable sustained phonation (CSP): Participants were instructed to take a deep breath, then to produce a sustained open vowel $(/ \mathrm{a} /)$ at a comfortable pitch and loudness for at least $5 \mathrm{~s}$ once data capture was initiated.

(c) Voicing efficiency: Participants were instructed to repeat the voiced vowel /a/ and the voiceless stop plosive /p/ nine times in vowel/consonant format (i.e., lapapapapapapapa/), placing equal stress on each syllable as described by Zraick, Smith-Olinde, and Shotts (2012). To ensure consistent rhythm, participants were trained on the speaking task until they produced the syllable trains evenly and at a comfortable loudness level.

Three trials of each of the foregoing tasks were conducted, and the average of the three trials was used for analysis. Participants were provided with instructions for each task before every trial. Mean phonatory airflow (liters per second) was derived from CSP and MSP tasks individually. CSP protocol is based on analysis of a sustained portion of voicing that is comfortable in pitch and loudness for the participant. We were, however, interested in the total expiratory volume and phonation time as well, and therefore the MSP protocol was also used. Estimates of subglottal pressure, mean phonatory sound pressure level, Rlaw and phonatory airflow were derived from the voicing efficiency task. Measures of average peak (intraoral) air pressure during adjacent productions of the consonant $/ \mathrm{p} /$ (across syllables 2-8) provided the estimate of subglottal pressure (Zraick et al., 2012). Mean airflow during voicing was derived from the oral airflow measures during the vowel segments. Two measures (peak air pressure and mean airflow during voicing) were subsequently used by 
the PAS software to calculate Rlaw value, which is defined as the ratio of peak pressure/airflow. The airflow signal was examined to ensure a baseline (zero) was reached for each pressure peak so as to not underestimate subglottic pressure.

Patient perception. All participants completed the 30-item Voice Handicap Index before and after the treatment. This provided the patient perceptions of the handicapping effects of their voice.

\section{Treatment}

Participants were randomly assigned to one of two groups. The random assignment of participants was carried out after the assessment session before initiation of any treatment. Participants in Group 1 received treatment in person at the medical center. Participants in Group 2 received treatment at one of 13 regional affiliated satellite clinics through an Internet protocol-based videoconferencing system supported by a Tandberg interactive audiovideo unit. The farthest satellite clinic was 190 miles from the on-site clinic, and the closest was 1 mile. Participants in Group 2 traveled not more than 15 miles to the satellite clinic. Participants in Group 2 signed an additional form granting permission to be photographed and video recorded. The clinician worked with the patient from the host medical center. The participants in both groups attended 12 sessions of voice therapy across 6 weeks (two sessions per week). The number of sessions was initially established considering reimbursement that is currently available for speech-language therapy. Also, because each patient differs in terms of baselines, compliance, and general attitudes toward treatment, it is difficult to determine a specific number of sessions to be the standard for all patients. Six weeks of treatment have been targeted in previous studies on voice therapy (MacKenzie, Millar, Wilson, Sellars, \& Deary, 2001). McCrory (2001) conducted a retrospective audit of various parameters defining best practice in voice therapy and reported an average of two to 12 sessions of therapy for vocal fold nodules. Lockhart, Paton, and Pearson (1997) reported the average number of treatment sessions for vocal strain was two to 14 and about two to 15 for ventricular fold overaction in two different voice centers. Considering these factors, a uniform number of 12 sessions for all patients was determined. Additional research into the average number of treatment sessions necessary for varying pathologies and patterns of baseline measures is underway. All treatment sessions were conducted by the first author, who was, at the time, a doctoral student in communication sciences and disorders with a focus in voice disorders and dysphagia and with about 4 years' clinical experience in voice care.

\section{Part I: Vocal Hygiene (Approximately 10-15 Min)}

The protocol established by Nanjundeswaran et al. (2012) was used for vocal hygiene education. In Session 1, each participant completed a vocal hygiene questionnaire, which was reviewed with the participant. Participants were then provided instructions for improving vocal hygiene on the basis of their responses to the questionnaire. Vocal hygiene instructions included information on intake of appropriate fluids (noncaffeinated), controlled use of voice, and control of reflux or allergies if applicable. Recommendations were discussed, and problem areas were highlighted for each participant on the basis of clinical judgment. In each of the additional 11 sessions, participants conveyed the vocal hygiene instructions back to the clinician and discussed successes and problem areas since the previous session.

\section{Part II: Airflow Exercises (Flow Phonation; 20-25 Min per Session)}

Each treatment session typically used two of three airflow exercises: gargling, cup bubble blowing, and stretch and flow phonation, depending on the participant's success with each exercise. Each exercise uses a built-in form of biofeedback (water or tissue) and the same basic progression of activities: (a) airflow task without voicing to establish positive airflow; (b) adding voicing to the task; (c) moving up and down the pitch range during the voicing task; and (d) moving to a speaking/voicing task, removing biofeedback. During each vocalization attempt, the clinician listened for a clear and effortless vocal quality and trained the participant to listen and feel relaxation within the throat.

In greater detail, the gargling exercise required the participant to place a small amount of water in the mouth, recline the head, and gargle without voice 10 times for 5-6 s with breaks in between. The participant was instructed to relax the throat and gargle with enough airflow to make the bubbles pop up out of the mouth. After this was accomplished, the next step was to gargle the same way but with voice-again ensuring bubbles popped up out of the mouth. The third step was to gargle with the head back with the voice moving up and down pitch scales freely and relaxed. This was done 10 times as well. The fourth step began with the participant gargling with voice and then rolling the head forward while gargling, closing the mouth, and allowing the sound to come out the nares into a hum. The participant then swallowed the water, took an easy breath, and repeated "mmmmama mama," "mmmmay, may, may, may," and then continued with $/ \mathrm{m} /$ and other vowels. When voicing sounded sufficiently relaxed without laryngeal tension, it was carried over into words, such as mamma, marry, many, maybe, marble. This method was used to transition voice with gargling into voice without gargling and on into a more natural pattern of voice use. The focus was not about nasal sounds or frontal focus as much as moving the hum, which occurred from the head roll, into speech.

The cup bubble blowing exercise required the participant to take a clear plastic cup of water filled about two thirds of the way up, place the mouth over the cup, and tip it up until the top lip was in the water. The participant drew in a breath and blew bubbles without using voice, again to establish positive airflow. Bubbles were supposed to be actively popping up from the cup. This was done 10 times, and then voicing was added for 10 trials to make a "motorboat" sound. When voicing was added, bubbles 
were to remain as active as they were without the voice. In the third step, the participant blew bubbles with voice moving freely and relaxed up and down in pitch. In the fourth step, the participant began by blowing bubbles with the voice and then slowly pulled the cup away from the mouth. As the cup was pulled away, the participant maintained pursed lips and continued voicing with a relaxed, breathy "oooh." This step was repeated 10 times. For all the trials, the clinician provided feedback on vocal quality and perceived relaxation in the voice.

The final exercise involved stretch and flow phonation. For this exercise, the participant took a piece of tissue paper, separated the layers, and folded one layer in half. The tissue was held between the index and middle fingers near the top of the tissue, and the tissue was held in front of the face hanging where the mouth is centered. In the first part of the exercise, the participant blew air into the tissue such that the tissue moved back parallel to the floor for 4-5 s. This was done 10 times with breaks in between. Then the participant began as in Step 1, blowing air into the tissue. When the tissue was parallel to the floor, the participant added voice. The tissue was to remain parallel to the floor, so the voice would be very breathy. This was done 10 times with sufficient breaks. In Step 3, the participant began blowing air into the tissue and voicing simultaneously, ensuring it was parallel to the floor, and then said "one" with the same easy, breathy voice. This was repeated for numbers 2,3 , and so forth, up to 10 . The fourth step was done the same way but with "H" and "WH" initiated phrases rather than words (i.e., "How are you?" "What time is it?").

Participants started with gargling and cup bubble blowing. As they mastered one or both of these exercises, they were moved into stretch and flow exercises, typically in the fifth session for 13 patients and the seventh session for one patient who took longer to master relaxation with the former exercises. The sessions, including vocal hygiene reviews and voice work, typically lasted for about $45 \mathrm{~min}$.

\section{Telepractice Component}

A hybrid approach was used in the telepractice group; that is, all the participants were evaluated in person during the pre- and posttreatment sessions, but treatment was delivered using telepractice. Because the primary aim of the study was to determine the use of telepractice for a therapeutic activity and because participants were not randomized to the groups until after initial assessment, pre- and posttreatment evaluations were carried out in person. The treatment was delivered using a Tandberg interactive video-audio unit. As noted earlier, all sessions for both groups of participants were delivered by one clinician (the first author). Care was taken to ensure a quiet environment, and confirmation was sought from participants to ensure they could see and hear the clinician before each session was begun. The clinician also made sure the positioning of the participant was appropriate for adequate visualization of breathing patterns, and assessment of each treatment exercise. Support staff in each center ensured that the camera and microphone were positioned appropriately. Staff also provided materials needed for treatment, such as a clear cup, water, and tissue papers. Information technology support staff were accessible for all 12 treatment sessions for each participant in case of technical difficulties. There was one instance of a delayed connection during the sixth session for Participant TVS022 and another instance of poor audio and reverberation in the first session for Participant TVS003. The information technology support staff resolved these issues quickly. The treatment was delivered the same way for both in-person and telepractice groups.

All 14 participants completed the entire research protocol. All 14 participants underwent a pretreatment assessment within 2 weeks of a diagnosis of MTD and a posttreatment assessment within 5 days of completion of treatment.

\section{Statistical Analysis of Data}

Acoustic measures of VTI and NHR, aerodynamic measures of mean airflow during CSP and MSP tasks, aerodynamic resistance, overall severity of voice quality as measured by the CAPE-V, and the total Voice Handicap Index score were subjected to separate statistical analyses using SPSS Version 20. In an ideal situation, we would have performed two-way analyses of variance for withinparticipant (time point) and between-participant (treatment delivery mode) variables. However, our sample size of only seven per group would have posed threats to interpretation of the data. The likelihood of both underpowered analyses and violations of fundamental assumptions of the distribution of the data was high, and therefore nonparametric analyses were performed as follows.

\section{Analysis 1: Telepractice Versus In Person}

To analyze the outcome of treatment across the two groups, a change variable was first derived. For example, ChangeNHR was calculated by subtracting posttreatment NHR values from pretreatment NHR values (NHRPre NHRPost). A change variable was similarly obtained for all acoustic, aerodynamic, auditory-perceptual, and selfperception of voice handicap measures. The change variable of each measure was then subjected to Mann-Whitney $U$ test to examine if a change in a variable as an effect of treatment is comparable across the two treatment delivery modes.

\section{Analysis 2: Pretreatment Versus Posttreatment}

The Wilcoxon signed-ranks test was performed to examine the magnitude and significance of changes in outcome measures after treatment. Actual pretreatment and posttreatment measures (and not change in a measure) were compared across the two time points. Additionally, Cohen's $d$ measures as part of effect sizes of treatment were computed. Marginal means of change in outcome measures from before versus after treatment were also calculated. 


\section{Results \\ Reliability of Perceptual Voice Ratings}

The data corpus was rated for vocal quality by the second author, and $10 \%$ of the data were rerated by two other speech-language pathologists (the fourth author and another speech-language pathologist) on the basis of audio recordings using the CAPE-V, which in turn uses a $100-\mathrm{mm}$ visual analog scale. Pre- and posttreatment samples of each participant's voice were provided to each rater with order of occurrence randomized. Thus, each rater had two samples to listen to and rate without the knowledge of whether it had been obtained prior to or after treatment. The .nsp files obtained from the CSL program were converted to wave files (.wav) and were presented through a computer program (Windows 7 Media Player) through high-fidelity headphones. The judges completed voice quality ratings in one session. Because the data are continuous, intraclass correlations were calculated to measure interrater reliability of ratings. Intraclass correlation values for overall severity were high, on the order of .92 for pretreatment samples and .84 for posttreatment samples, demonstrating a strong interrater reliability of ratings.

\section{Telepractice Versus in Person (Across Groups)}

The Mann-Whitney $U$ test indicated that outcome measures for change in CAPE-V overall severity $(U=15$, $p=.62)$, change in NHR $(U=14, p=.21)$, change in VTI $(U=19, p=.84)$, change in mean airflow for $\operatorname{MSP}(U=$ $8.5, p=.073)$, change in mean airflow for CSP $(U=11$, $p=.181)$, change in Rlaw $(U=11, p=.181)$, and change in Voice Handicap Index scores $(U=20, p=.259)$ did not differ significantly between the groups (see Table 3 ). This is further depicted in Figures 1-5, which demonstrate the direction of change of marginal means in the two groups from pretreatment to posttreatment. Graphical depictions of the data (see Figures 1-5) indicate that effects of treatment on both groups appear visually comparable, and most changes posttreatment occurred in the same direction between in-person and telepractice groups (see Figures 1-5). The one exception was mean airflow for voicing (see Figure 4). Mean airflow during MSP and CSP tasks increased as an effect of treatment in the telepractice group and decreased as an effect of treatment in the in-person group, although the change was not statistically significant.
Pretreatment Versus Posttreatment (Across Time Points)

The Wilcoxon signed-ranks test yielded statistically significant improvements in the CAPE-V (clinician perception of severity; $Z=3.30, p=.001$ ), Voice Handicap Index (patient perception of handicap; $Z=3.17, p=.002$ ), preto posttherapy, and some aerodynamic measures: mean airflow during $\operatorname{CSP}(Z=2.04, p=.04)$ and $\operatorname{MSP}(Z=$ 2.12, $p=.034)$, on the basis of norms reported by Zraick et al. (2011; see Table 4). Changes in acoustic measures of NHR $(Z=1.92, p=.055)$ and VTI $(Z=1.78, p=.075)$ and the aerodynamic measure of laryngeal airway resistance (Rlaw; $Z=1.01, p=.311$ ) moved in the same direction of a therapeutic effect pre- to posttherapy (see Figures 1-5) for the in-person and telepractice groups, demonstrating clinical improvement, but did not reach statistical significance. In addition, Cohen's $d$ effect sizes were computed for each outcome measure. The effect sizes for CAPE-V severity, NHR, VTI, mean airflow in CSP, mean airflow in MSP, Rlaw, and VHI were 1.73, 0.59, 0.82, 0.29, $0.27,0.41$ and 1.24 , respectively. It is important to observe that there were differences in baseline values of phonatory airflow measures across participants.

\section{Discussion}

The primary purpose of this study was to determine whether flow phonation treatment for primary MTD could be delivered equally well via telepractice and in-person treatment. Results of across-groups comparison yielded no significant differences, demonstrating that participants with primary MTD enrolled randomly in telepractice sessions performed similarly to participants enrolled in in-person treatment. It was also important to determine whether the treatment approaches were similarly effective or ineffective; that is, it is possible that neither group improved, or both did, or even got worse. Considering the across-groups and across-time points analyses, the quality of voicing, as judged by the clinician (CAPE-V) and the patient's perception of handicap (Voice Handicap Index) improved, again, irrespective of the treatment delivery mode. The majority of participants also improved on acoustic and aerodynamic measures regardless of the method of delivery, although improvements were not statistically significant.

These results are consistent with earlier investigations supporting the use of telepractice in delivering voice

Table 3. Results of a Mann-Whitney $U$ test for auditory-perceptual, acoustic, aerodynamic, and quality-of-life measures across the two groups (telepractice vs. in person).

\begin{tabular}{|c|c|c|c|c|c|c|}
\hline $\begin{array}{l}\text { Change in } \\
\text { CAPE-V severity }\end{array}$ & $\begin{array}{l}\text { Change } \\
\text { in NHR }\end{array}$ & $\begin{array}{l}\text { Change } \\
\text { in VTI }\end{array}$ & $\begin{array}{l}\text { Change in mean } \\
\text { airflow in CSP }\end{array}$ & $\begin{array}{l}\text { Change in mean } \\
\text { airflow in MSP }\end{array}$ & $\begin{array}{l}\text { Change } \\
\text { in Rlaw }\end{array}$ & $\begin{array}{c}\text { Change } \\
\text { in VHI }\end{array}$ \\
\hline $\begin{array}{l}U=15 \\
p=.620\end{array}$ & $\begin{array}{l}U=14 \\
p=.209\end{array}$ & $\begin{array}{l}U=19 \\
p=.836\end{array}$ & $\begin{array}{l}U=11 \\
p=.181\end{array}$ & $\begin{array}{l}U=8.5 \\
p=.073\end{array}$ & $\begin{array}{l}U=11 \\
p=.181\end{array}$ & $\begin{array}{l}U=20 \\
p=.259\end{array}$ \\
\hline
\end{tabular}

Note. $\quad \mathrm{CAPE}-\mathrm{V}=$ Consensus Auditory Perceptual Evaluation of Voice; NHR = noise-to-harmonic ratio; VTI = voice turbulence index; CSP = comfortable sustained phonation; MSP = maximum sustained phonation; Rlaw = laryngeal resistance; VHI = Voice Handicap Index. 
Figure 1. Marginal means of the Consensus Auditory Perceptual Evaluation of Voice (CAPE-V) severity for the treatment groups at two time points 1 and 2 (pre- and posttreatment, respectively).

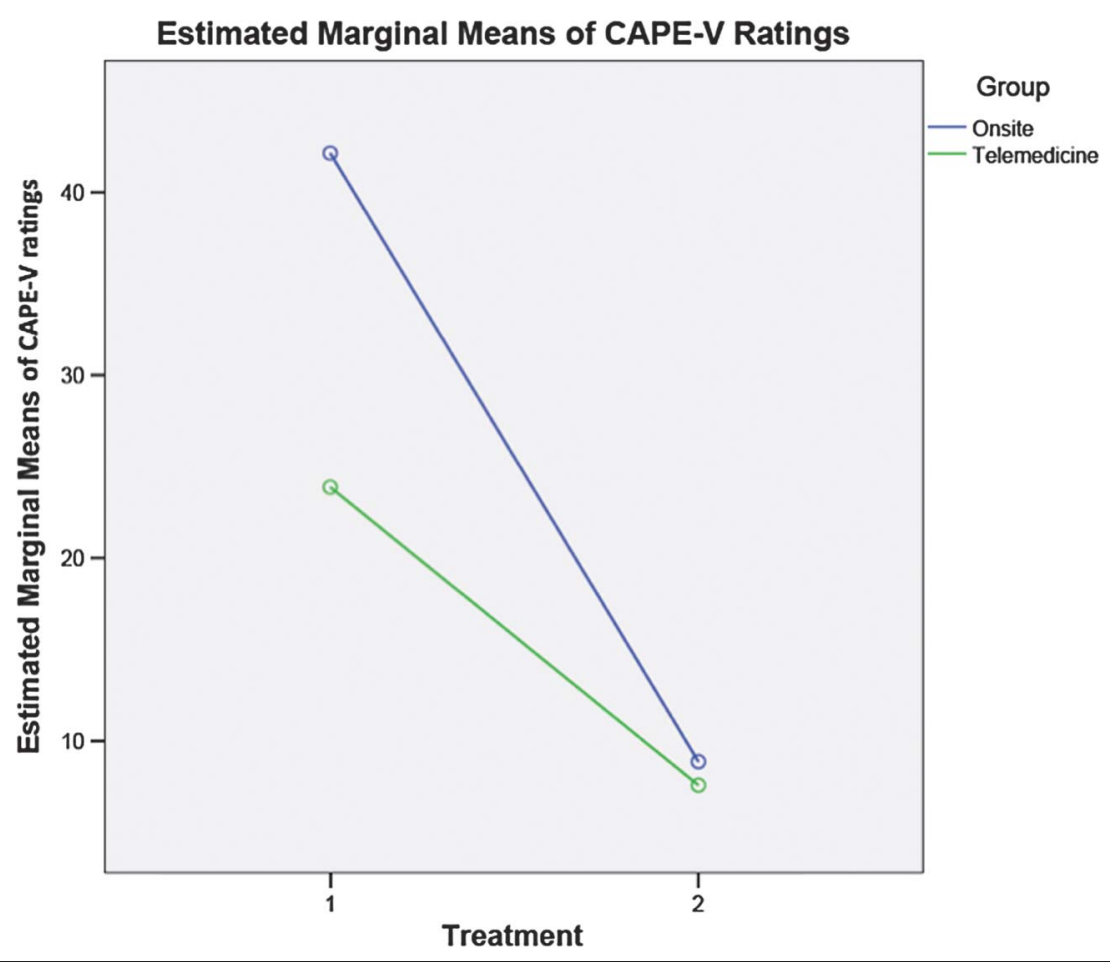

therapy. To be specific, the results are in general agreement with those from studies by Mashima et al. (2003), Tindall et al. (2008), and Constantinescu et al. (2010a, 2010b, 2011), all of whom reported positive outcomes of delivering voice treatment for different pathologies through telepractice.
It is important to clarify the types of treatment used when investigating telepractice given that telepractice is not a treatment but rather a method of delivering treatment. Different treatments may be more suited for telepractice than others. Although it may not be necessary to provide data supporting

Figure 2. Marginal means of noise-to-harmonic ratio (NHR) and voice turbulence index (VTI) from multidimensional voice profile for the treatment groups at two time points (pre- and posttreatment). The $y$-axis has no units because the measures indicate a ratio.

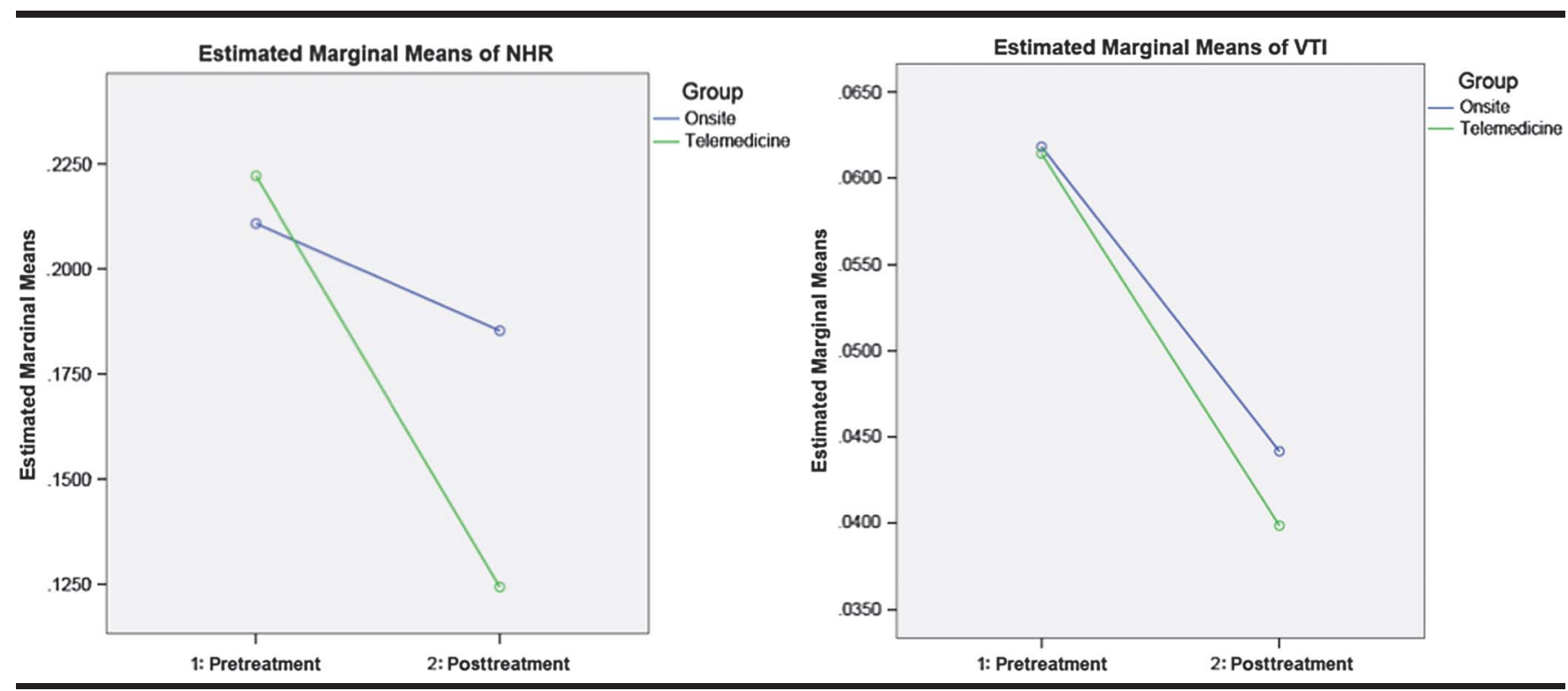


Figure 3. Marginal means for laryngeal resistance for the treatment groups at two time points (pre- and posttreatment).

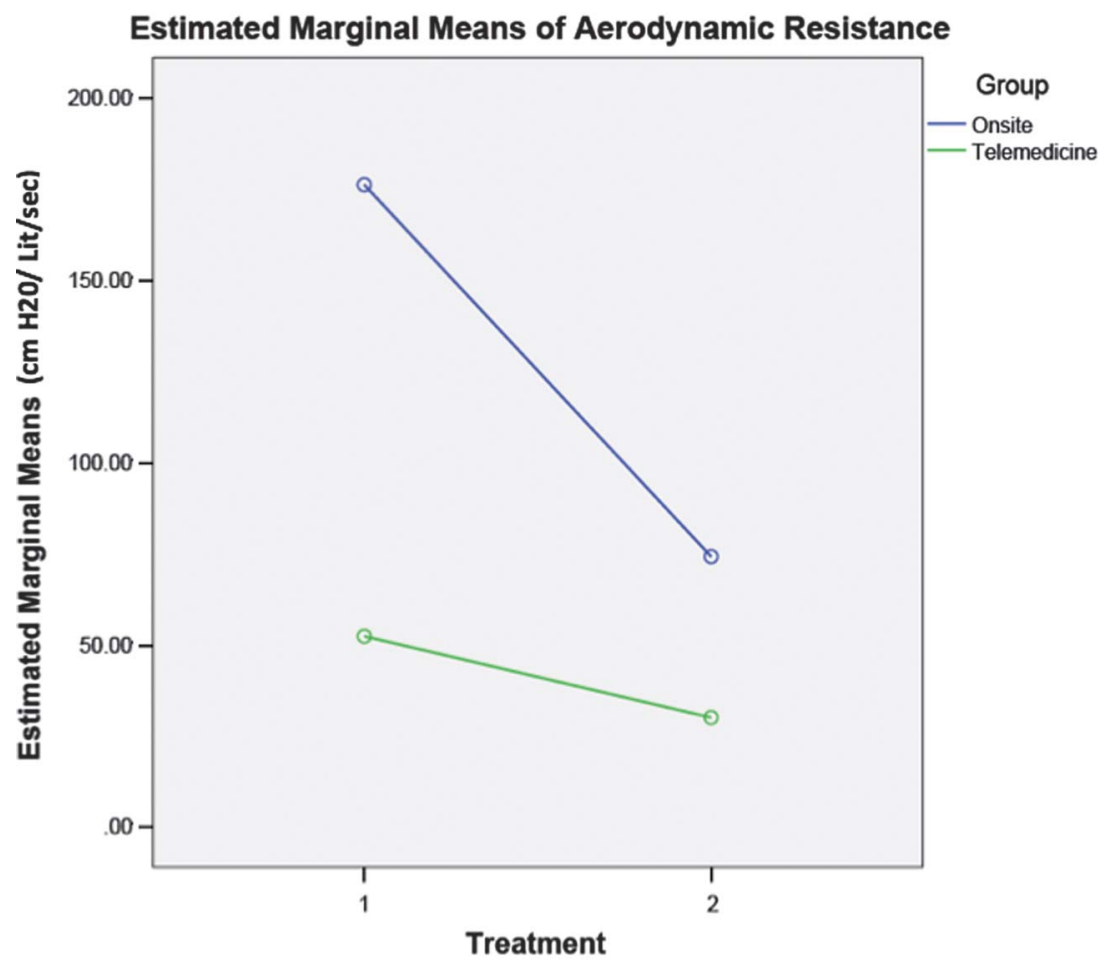

every type of voice therapy for use with telepractice, a relatively small number of studies using voice treatments have been published, and thus far, none have dealt with MTD. The current data support the use of telepractice for delivering voice therapy for treating primary MTD with flow phonation exercises. The impact of the results of this study could be very positive for many individuals who suffer this disorder and might not otherwise receive adequate care for the problem. The goal of the speech-language pathologist is to maximize functional abilities and promote quality of

Figure 4. Marginal means of mean airflow during comfortable (CSP) and maximum (MSP) sustained phonation for the treatment groups at two time points (pre- and posttreatment). $\mathrm{sec}=$ second.
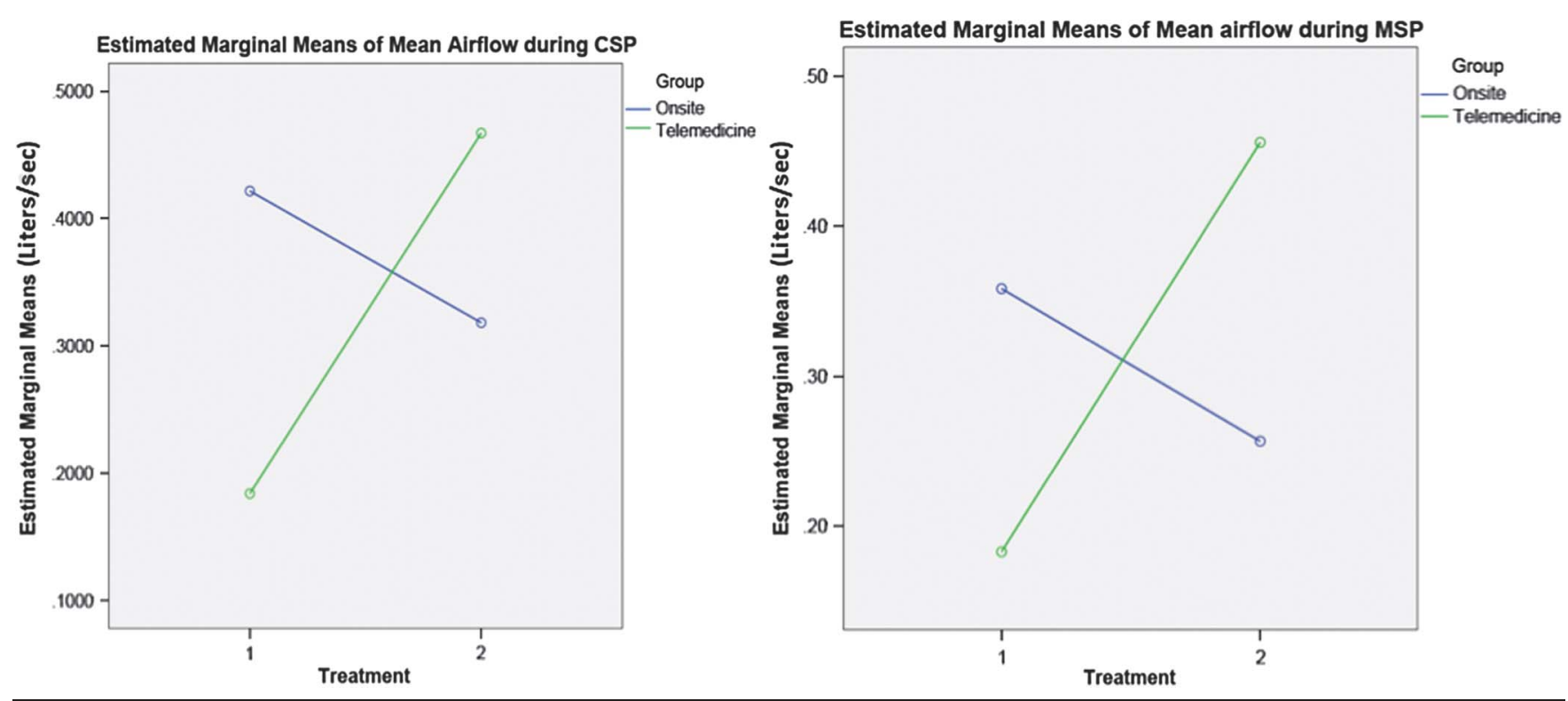
Figure 5. Marginal means for ratings on the Voice Handicap Index (VHI) for the treatment groups at two time points (pre- and posttreatment).

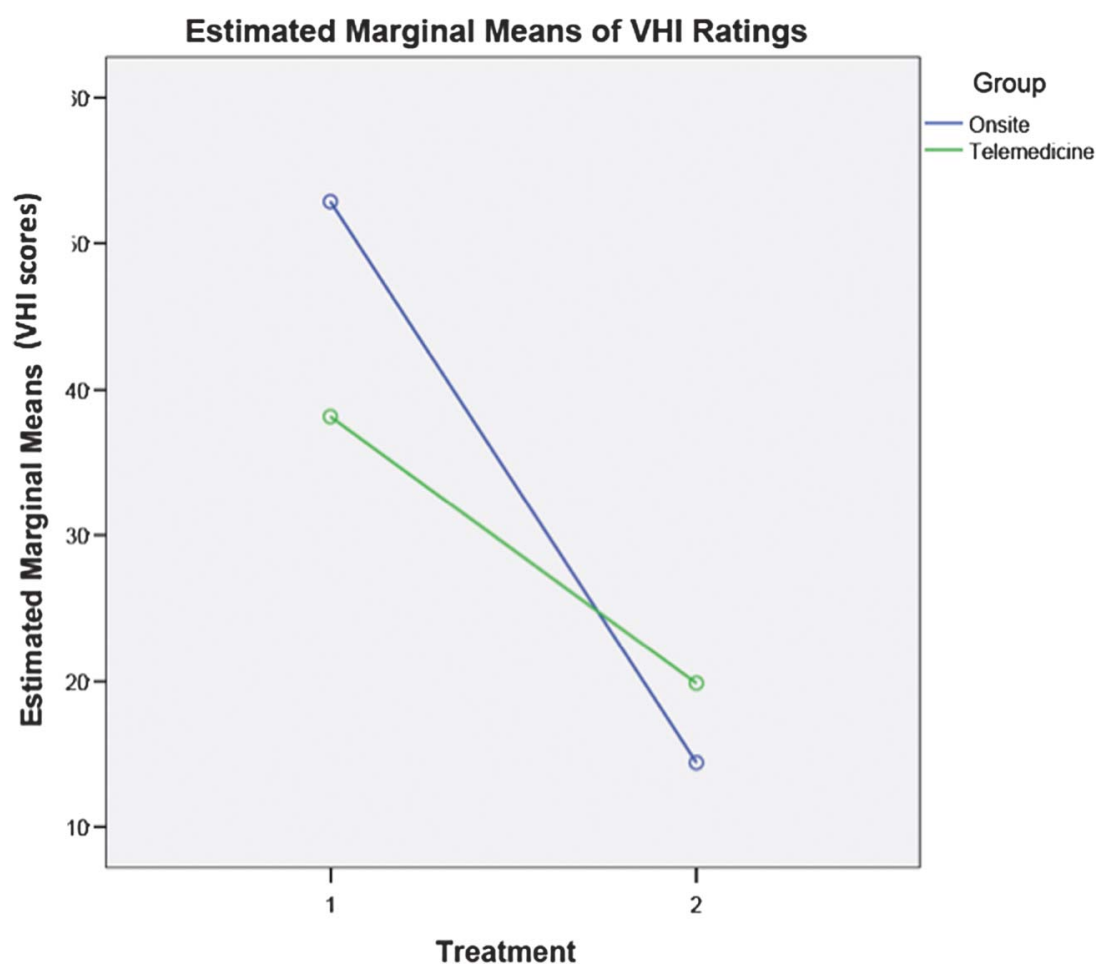

life for individuals with communication disorders. Some individuals live in rural, underserved areas far from a medical center or a health facility that offers speech pathology services. Others have physical impairments (e.g., mobility issues) that prevent them from going somewhere to receive treatment. Still others may have no means of transportation. Telepractice is one tool that may help reach such individuals, and the results of this study suggest that telepractice can, for certain disorders and treatments, be as beneficial as treating patients for their vocal symptoms in person. Moreover, it can reduce or eliminate travel time and inconveniences associated with travel as well as costs, and it provides a "greener" option for those who live far away, with less of a negative impact on our planet.

The results also shed some light on specific outcomes of the flow phonation exercises and why a benefit was observed. Phonatory airflow is often decreased when laryngeal tension creates a constriction of the glottis. Reducing the tension should then open the glottis and allow for increased phonatory airflow. Phonatory airflow might also be increased in some patients with MTD because tension, especially anteriorposterior tension, may inhibit glottal closure. Thus, when airflow is too high, normalization would mean that it is reduced. When airflow is too low normalization would mean it is increased. Phonatory airflow increased more in the telepractice group than the in-person group, but individuals in the inperson group started with a higher mean phonatory airflow, indicating less of a need to increase it. The difference in this particular measure, therefore, does not indicate one group did better than the other but rather that the groups had some differences in the effects of putative muscle tension on the coordination of the respiratory and phonatory systems. This premise has been documented in studies by Higgins et al. (1999) and Gillespie et al. (2013), who have reported wide

Table 4. Results of a Wilcoxon signed-ranks test for auditory-perceptual, acoustic, aerodynamic, and quality-of-life measures across the two time points (pre- and posttreatment).

\begin{tabular}{|c|c|c|c|c|c|c|}
\hline CAPE-V severity & NHR & VTI & Mean airflow in CSP & Mean airflow in MSP & Rlaw & VHI \\
\hline $\begin{array}{l}Z=-3.297 \\
p=.001^{*}\end{array}$ & $\begin{array}{l}Z=-1.922 \\
p=.055^{\star \star}\end{array}$ & $\begin{array}{l}Z=-1.779 \\
p=.075^{\star \star}\end{array}$ & $\begin{array}{l}Z=-2.040 \\
p=.041^{*}\end{array}$ & $\begin{array}{l}Z=-2.119 \\
p=.034^{*}\end{array}$ & $\begin{array}{l}Z=-1.013 \\
p=.311\end{array}$ & $\begin{array}{l}Z=-3.170 \\
p=.002^{*}\end{array}$ \\
\hline
\end{tabular}

Note. $\mathrm{CAPE}-\mathrm{V}=$ Consensus Auditory Perceptual Evaluation of Voice; NHR = noise-to-harmonic ratio; VTI = voice turbulence index; CSP = comfortable sustained phonation; MSP = maximum sustained phonation; Rlaw = laryngeal resistance; VHI = Voice Handicap Index.

${ }^{*} p<.05 .{ }^{* *}$ Approaching significance at $p<.05$. 
variability in aerodynamic characteristics in participants with MTD and different aerodynamic subtypes in participants with primary MTD, respectively. The fact that all patients improved on perceptual measures of voice by the clinician as well as self-perceived voice handicap indicate the direction and amount of the changes in airflow were likely beneficial. This method of treatment was designed to increase airflow in patients with severely limited flow of air, but our results suggest it may also be beneficial for patients with somewhat greater airflow initially. A closer examination of Rlaw could shed additional light on these findings, although differences in Rlaw were present before treatment across the two groups in our study, limiting further interpretation. Additional research should be conducted to determine whether levels of airflow and Rlaw may predict who would benefit most from this treatment method.

It is important to note that these findings are specific to primary MTD alone. As noted earlier, primary MTD is a stand-alone laryngeal tension abnormality causing changes to Rlaw and consequently airflow. The patterns of airflow and Rlaw impairments could be significantly different in cases of secondary MTD that are concurrent with another physical impairment, such as spasmodic dysphonia or laryngopharyngeal reflux disorders. Therefore, the results have to be interpreted in view of primary MTD alone.

There are a number of weaknesses in this study. Our sample size was particularly small. We determined sample size on the basis of statistical results from pilot data (McCullough et al., 2012), but those data were focused on the exercises themselves and not the use of telehealth technology. As stated, larger numbers guided by detailed power analyses are needed to more critically examine different types of MTD and changes in Rlaw in relation to phonatory airflow and tease out the effects of initial severity. More data are needed and are in progress on dose response as 12 sessions may be necessary for some whereas only six or fewer may be necessary for others. Procedural reliability was not measured in our study. Although only one clinician conducted all treatment sessions for all the study participants, it would have been useful to ensure uniformity of treatment by objective procedural fidelity reports. Percentage agreement could have been a better measure to report reliability of auditory perceptual measures. We used intraclass correlations, which may not reflect best estimates of interjudge reliability for perceptual analysis of voice in particular (Kreiman, Gerratt, Kempster, Erman, \& Berke, 1993). Treatment compliance was generally quite strong, but data regarding some variations are currently being analyzed. We also used change scores as a dependent variable for our analyses. The use of normalized raw scores may have altered or assisted with interpretation to some degree. An important aspect that could have offered clearer insights into the results is the differences in baseline values of various airflow measures. Although control for participant selection bias was exercised using randomization, it also led to heterogeneity across the groups with respect to airflow measures. Future randomized controlled studies on treatment issues such as this should ensure some sort of stratification on the basis of baseline severity.

\section{Conclusions}

The specific aim of this study was to explore the use of telepractice in delivering flow phonation exercises for primary MTD. MTD is one of the most common behavioral voice disorders seen in clinics, and data supporting the use of telepractice for providing behavioral interventions to individuals with this form of dysphonia are scarce. These data support the use of telepractice to deliver this treatment successfully and may improve patient care by providing treatment to underserved individuals in rural or other populations without the ability to come to medical centers where such treatment is available.

\section{Acknowledgments}

This study was funded by the Center for Distance Health, University of Arkansas for Medical Sciences, Little Rock. We thank Amanda Davis for her help with the perceptual voice analysis.

\section{References}

Altman, K. W., Atkinson, C., \& Lazarus, C. (2005). Current and emerging concepts in muscle tension dysphonia: A 30-month review. Journal of Voice, 19, 261-267.

American Speech-Language-Hearing Association. (2002). Survey of telepractice use among audiologists and speech-language pathologists. Rockville, MD: Author.

American Speech-Language-Hearing Association. (2005). ASHA speech-language pathology health care survey issue briefs: Vacancies. Rockville, MD: Author.

American Speech-Language-Hearing Association. (2009). 2009 SLP health care survey summary report: Number and type of responses. Rockville, MD: Author.

Awan, S. N., \& Roy, N. (2009). Outcomes measurement in voice disorders: Application of an acoustic index of dysphonia severity. Journal of Speech, Language, and Hearing Research, 52, 482-499.

Bhuta, T., Patrick, L., \& Garnett, J. D. (2004).Perceptual evaluation of voice quality and its correlation with acoustic measurements. Journal of Voice, 18, 299-304.

Boone, D. R., McFarlane, S. C., Von Berg, S. L., \& Zraick, R. I. (2013). The voice and voice therapy (9th ed.). Boston, MA: Allyn \& Bacon.

Cohen, S. M., Kim, J., Roy, N., Asche, C., \& Courey, M. (2012a). Direct health care costs of laryngeal diseases and disorders. The Laryngoscope, 122, 1582-1588.

Cohen, S. M., Kim, J., Roy, N., Asche, C., \& Courey, M. (2012b). Prevalence and causes of dysphonia in a large treatment-seeking population. The Laryngoscope, 122, 343-348.

Colton, R. H., \& Casper, T. K. (1990). Understanding voice problems: A physiological perspective for diagnosis and treatment (2nd ed.). Baltimore, MD: Williams \& Wilkins.

Constantinescu, G., Theodoros, D., Russell, T., Ward, E., Wilson, S., \& Wootton, R. (2010a). Assessing disordered speech and voice in Parkinson's disease: A telerehabilitation application. International Journal of Language \& Communication Disorders, $45,630-644$ 
Constantinescu, G., Theodoros, D., Russell, T., Ward, E., Wilson, S., \& Wootton, R. (2011). Treating disordered speech and voice in Parkinson's disease online: A randomized controlled noninferiority trial. International Journal of Language \& Communication Disorders, 46, 1-16.

Constantinescu, G. A., Theodoros, D. G., Russell, T. G., Ward, E. C., Wilson, S. J., \& Wootton, R. (2010b). Home-based speech treatment for Parkinson's disease delivered remotely: A case report. Journal of Telemedicine and Telecare, 16, 100-104.

de Krom, G. (1995). Some spectral correlates of pathological breathy and rough voice quality for different types of vowel fragments. Journal of Speech, Language, and Hearing Research, 38, 794-811.

Di Nicola, V., Fiorella, M. L., Spinelli, D. A., \& Fiorella, R. (2006). Acoustic analysis of voice in patients treated by reconstructive subtotal laryngectomy: Evaluation and critical review. Acta Otorhinolaryngologica Italica, 26, 59-68.

Gartner-Schmidt, J. L. (2010). Flow phonation. In J. Stemple \& L. T. Fry (Eds.), Voice therapy: Clinical studies (3rd ed.; 84-92). San Diego, CA: Plural.

Gillespie, A. I., Gartner-Schmidt, J., Rubinstein, E. N., \& Abbott, K. V. (2013). Aerodynamic profiles of women with muscle tension dysphonia/aphonia. Journal of Speech, Language, and Hearing Research, 56, 481-488.

Hapner, E., Portone-Maira, C., \& Johns, M. M., III (2009). A study of voice therapy dropout. Journal of Voice, 23, 337-340.

Higgins, M. B., Chait, D. H., \& Schulte, L. (1999). Phonatory air flow characteristics of adductor spasmodic dysphonia and muscle tension dysphonia. Journal of Speech, Language, and Hearing Research, 42, 101-111.

Jacobson, B. H., Johnson, A., Grywalski, C., Silbergleit, A., Jacobson, G., Benninger, M. S., \& Newman, C. W. (1997). The Voice Handicap Index (VHI): Development and validation. American Journal of Speech-Language Pathology, 6, 66-70.

Kempster, G. B., Gerratt, B. R., Verdolini Abbott, K., BarkmeierKramer, J., \& Hillman, R. E. (2009). Consensus AuditoryPerceptual Evaluation of Voice: Development of a standardized clinical protocol. American Journal of Speech-Language Pathology, $18,124-132$.

Koufman, J. A., \& Blalock, P. D. (1982). Classification and approach to patients with functional disorders. Annals of Otology, Rhinology \& Laryngology, 91, 372-377.

Kotby, M. N. (1995). The accent method of voice therapy. San Diego, CA: Singular.

Kreiman, J., Gerratt, B. R., Kempster, G. B., Erman, A., \& Berke, G. S. (1993). Perceptual evaluation of voice quality review, tutorial, and a framework for future research. Journal of Speech, Language, and Hearing Research, 36, 21-40.

Lockhart, M. S., Paton, F., \& Pearson, L. (1997). Targets and timescales: A study of dysphonia using objective assessment. Logopedics Phoniatrics Vocology, 22, 15-24.

MacKenzie, K., Millar, A., Wilson, J. A., Sellars, C., \& Deary, I. J. (2001). Is voice therapy an effective treatment for dysphonia? A randomised controlled trial. British Medical Journal, 323, 658.

Mashima, P. A., Birkmire-Peters, D. P., Holtel, M. R., \& Syms, M. J. (1999). Telehealth applications in speech-language pathology. Journal of Healthcare Information Management, 13(4), 71 .

Mashima, P. A., Birkmire-Peters, D. P., Syms, M. J., Holtel, M. R., Burgess, L. P. A., \& Peters, L. J. (2003). Telehealth: Voice therapy using telecommunications technology. American Journal of Speech-Language Pathology, 12, 432-439.
Masino, C., Rubinstein, E., Lem, L., Purdy, B., \& Rossos, P. G. (2010). The impact of telemedicine on greenhouse gas emissions at an academic health science center in Canada. Telemedicine and e-Health, 16, 973-976.

Mathieson, L., Hirani, S. P., Epstein, R., Baken, J., Wood, G., \& Rubin, J. S. (2009). Laryngeal manual therapy: A preliminary study to examine its effects in the management of muscle tension dysphonia. Journal of Voice, 23, 353-366.

McCrory, E. (2001). Voice therapy outcomes in vocal fold nodules: A retrospective audit. International Journal of Language \& Communication Disorders, 36(Suppl. 1), 19-24.

McCullough, G. H., Zraick, R. I., Balou, S., Pickett, H. C., Rangarathnam, B., \& Tulunay-Ugur, O. E. (2012). Treatment of laryngeal hyperfunction with flow phonation: A pilot study. Journal of Laryngology and Voice, 2, 64-69.

Morrison, M. D., \& Rammage, L. A. (1993). Muscle misuse voice disorders: Description and classification. Acta Oto-Laryngologica, $113,428-434$.

Nanjundeswaran, C., Li, N. Y., Chan, K. M., Wong, R. K., Yiu, E. M. L., \& Verdolini-Abbott, K. (2012). Preliminary data on prevention and treatment of voice problems in student teachers. Journal of Voice, 26, 816.e1-816.e12.

Portone-Maira, C., Wise, J. C., Johns, M. M., III, \& Hapner, E. R. (2011). Differences in temporal variables between voice therapy completers and dropouts. Journal of Voice, 25, 62-66.

Ramig, L., Sapir, S., Fox, C., \& Countryman, S. (2001). Changes in vocal intensity following intensive voice treatment (LSVT) in individuals with Parkinson's disease: A comparison with untreated patients and normal age-matched controls. Movement Disorders, 16, 79-83.

Roy, N. (2003). Functional dysphonia. Current Opinion in Otolaryngology \& Head and Neck Surgery, 11, 144-148.

Roy, N. (2008). Assessment and treatment of musculoskeletal tension in hyperfunctional voice disorders. International Journal of Speech-Language Pathology, 10, 195-209.

Roy, N., Ford, C. N., \& Bless, D. M. (1996). Muscle tension dysphonia and spasmodic dysphonia: The role of manual laryngeal tension reduction in diagnosis and management. Annals of Otology, Rhinology \& Laryngology, 105, 851-856.

Roy, N., \& Leeper, H. A. (1993). Effects of manual laryngeal musculoskeletal tension reduction technique for functional voice disorders: Perceptual and acoustic measures. Journal of Voice, 7, 242-249.

Rubin, J., Sataloff, R., \& Korovin, G. (2006). Diagnosis and treatment of voice disorders (3rd ed.). San Diego, CA: Plural.

Sama, A., Carding, P. N., Price, S., Kelly, P., \& Wilson, J. A. (2001). The clinical features of functional dysphonia. The Laryngoscope, 111, 458-463.

Stemple, J. C., Glaze, L. E., \& Gerdeman, B. K. (2000). Clinical voice pathology: Theory and management ( 2 nd ed.). Boston, MA: Cengage Learning.

Stone, R. E., \& Casteel, R. L. (1982). Intervention in non-organically based dysphonia. In M. Filter (Ed.), Phonatory disorders in children (pp. 132-165). New York, NY: Charles C Thomas.

Tindall, L. (2012). The use of telepractice technology to provide speech and language services to persons aging with communication disorders. SIG 15 Perspectives on Gerontology, 17, 94-102.

Tindall, L. R., Huebner, R. A., Stemple, J. C., \& Kleinert, H. L. (2008). Videophone-delivered voice therapy: A comparative analysis of outcomes to traditional delivery for adults with Parkinson's disease. Telemedicine and e-Health, 14, 1070-1077. 
Van Houtte, E., Van Lierde, K., \& Claeys, S. (2011). Pathophysiology and treatment of muscle tension dysphonia: A review of the current knowledge. Journal of Voice, 25, 202-207.

Verdolini, K., Rosen, C., \& Branski, R. C. (Eds.). (2006). Classification manual for voice disorders-I. Mahwah, NJ: Erlbaum.

Verdolini-Marston, K., Burke, M., Lessac, A., Glaze, L., \&

Caldwell, E. (1995). Preliminary study of two methods of treatment for laryngeal nodules. Journal of Voice, 9, 74-85.
Zraick, R. I., Kempster, G. B., Connor, N. P., Thibeault, S., Klaben, B. K., Bursac, Z., ... Glaze, L. E. (2011). Establishing validity of the Consensus Auditory-Perceptual Evaluation of Voice (CAPE-V). American Journal of Speech-Language Pathology, 20, 14-22.

Zraick, R. I., Smith-Olinde, L., \& Shotts, L. L. (2012). Adult normative data for the KayPENTAX Phonatory Aerodynamic System Model 6600. Journal of Voice, 26, 164-176. 